\title{
The Effect to Single-Phase Load and Countermeasures of Zero Line Disconnection
}

\author{
J.F. Huang, Q.S. Chen, G.Y. Chen \\ Power Grid Co., Ltd. Foshan Chancheng District Power \\ Supply Bureau \\ China
}

\author{
W.C. Wen, H.P. Liang, Q.L. Tang \\ Foshan Electric Power Design Institute Co, Ltd \\ China
}

\author{
X. Cao \\ Department of Electric Engineering \\ North China Electric Power University \\ China
}

\begin{abstract}
The characteristic of load voltage and zero line voltage is studied when the zero line disconnection occurs in three-phase distribution system. The theoretical analysis show that: zerosequence voltage is appeared at the neutral point when the zero line disconnection occurs, and the size of zero-sequence voltage is relation to the load unbalance. The greater the load unbalance is, the greater the zero-sequence voltage. Based on this characteristic, a new methods to detect zero line disconnection is presented based on zero sequence voltage with zero line current blocking, an example prove that the method is feasible.
\end{abstract}

Keywords-three-phase distribution system; zero line disconnection; load unbalance; zero-sequence voltage

\section{INTRODUCTION}

Distribution system widely uses three phase four wire system [1] (TN - C system) or three-phase five wire (TN - S system). When the zero line disconnection occurs in threephase distribution system[2-4], an displacement voltage will be appeared at the neutral point of client, which will burn equipment in a large area and cause great economic losses to users; even may cause accident of human electric shock. Therefore, it is quite necessary to detect zero line disconnection and take corresponding measures.

The detection methods of zero line disconnection in distribution system are mainly based on phase-zero voltage and zero-ground voltage [5-7]. Phase-zero voltage method use the characteristic of the voltage increase at the phase with heavy load and the voltage reduce at the phase with light load. The principle of the above method is simple, but it may maloperate caused by the most load unbalance. A method to identify zero line disconnection based on DC injecting signal is presented [8-9]. The method of using additional injection signal to detect zero line disconnection will not be affected by the unbalance of loads, but the injection DC signal is a disturbance to load, and under the condition of symmetrical load operation, the zero line disconnection does not affect the load.

In this paper, the characteristic of load voltage and zero line voltage is studied when the zero line disconnection occurs in three-phase distribution system. And a new method based on zero sequence differential voltage with zero line current blocking to detect zero line disconnection is presented.

\section{THEORETICAL ANALYSIS}

Distribution system is TN system. Assuming that the threephase load is unbalanced, the load of phase $\mathrm{A}$ is the least, the load of phase B is larger and s the load of phase $\mathrm{C}$ is the most. When the zero line disconnection occurs at different positions, the equivalent circuit is shown as Figure. 1.

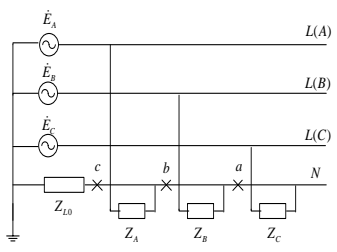

FIGURE I. EQUIVALENT CIRCUIT OF ZERO LINE .

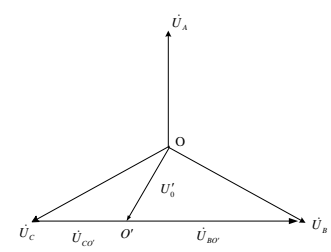

FIGURE II. VOLTAGE PHASOR DIAGRAM OF DISCONNECTION IN DISTRIBUTION SYSTEM ZERO LINE DISCONNECTION OCCURS AT POINT B.

(1) zero line disconnection occurs at point "a”, after which only single-phase load remains

When zero line disconnection occurs at point"a", the voltage of phase line and zero line of phase $C$ is equal, but with no current through load C, the load can not work properly.

(2) zero line disconnection occurs at point "b", after which double phases load remains

When zero line disconnection occurs at point"b", it has no effect on the load of phase A. The single-phase load of phase 
$\mathrm{B}$ and phases $\mathrm{C}$ which is after the disconnection is correspondently connect in series with the line to line voltage.

To the load of phase $\mathrm{B}$ and $\mathrm{C}$, the currents is: $\dot{I}=\frac{\dot{E}_{B C}}{Z_{B}+Z_{C}}$

The Voltage of Load B: $\dot{U}_{B O^{\prime}}=\dot{I} Z_{B}=\frac{\dot{E}_{B C}}{Z_{B}+Z_{C}} Z_{B}$

The Voltage of Load C: $\dot{U}_{C O^{\prime}}=\dot{I} Z_{C}=\frac{\dot{E}_{B C}}{Z_{B}+Z_{C}} Z_{C}$

When there are double phase load after the zero line disconnection, the voltage phasor diagram is shown as Figure.2. The Figure. 2 shows that: if the load of Phase B and $\mathrm{C}$ is equal, the load voltage of phase $\mathrm{B}$ and $\mathrm{C}$ is equal, which is equal to half of the line to line voltage, that is $\dot{U}_{B}^{\prime}=\dot{U}_{C}^{\prime}=\frac{\dot{E}_{B C}}{2}=190$. Else if the load of Phase B and $\mathrm{C}$ is unbalance, the larger the load is, the lower the voltage.

(3) zero line disconnection occurs at point "c", after which three phases load remains

when the zero line disconnection occurs at point " $c$ ", since there is no zero line to conduct imbalance current, an unbalance voltage will appeared at the neutral point to maintain the three-phase current vector sum to be equal to zero, which leads to the imbalance of three phase voltages. The equivalent circuit is shown as Figure 3.

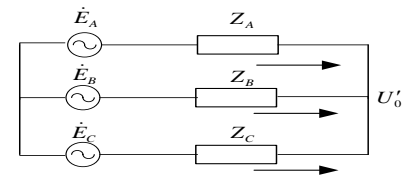

FIGURE III. EQUIVALENT CIRCUIT OF ZERO LINE DISCONNECTION OCCURS AT POINT C.

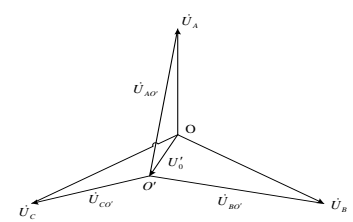

FIGURE IV. VOLTAGE PHASOR DIAGRAM OF ZERO LINE DISCONNECTION OCCURS AT POINT C.

By Kirchhoff's law:

$$
\frac{\dot{E}_{A}-U_{0}^{\prime}}{Z_{A}}+\frac{\dot{E}_{B}-U_{0}^{\prime}}{Z_{B}}+\frac{\dot{E}_{C}-U_{0}^{\prime}}{Z_{C}}=0
$$

The neutral point unbalance voltage is as follows:

$$
U^{\prime}=\left(\frac{\dot{E}_{A}}{Z_{A}}+\frac{\dot{E}_{B}}{Z_{B}}+\frac{\dot{E}_{C}}{Z_{C}}\right) /\left(\frac{1}{Z_{A}}+\frac{1}{Z_{B}}+\frac{1}{Z_{C}}\right)
$$

When there are three phase load after the zero line disconnection, the voltage phasor diagram is shown as Figure.4. From Figure. 4 we can see that: when the three-phase load is umbalance, an unbalance voltage will appeared at the neutral point to maintain the three-phase current vector sum to be equal to zero. The displacement voltage magnitude is only relationed to the load umbalance, which makes the neutral moves to the phase with the larger load, resulting in an unblance of three phase voltage. That is: the larger the load is, the lower the voltage is; and the more the load unbalance is, the greater the displacement voltage of neutral point is.

\section{THE EXAMPLE CALCULATION}

Distribution system is TN system. The rated voltage is $400 \mathrm{~V}$, transformer capacity is $400 \mathrm{kVA}$. The phase line is copper cable with the cross is $240 \mathrm{~mm}^{2}$, and power supply radius is 250m. By "GB/T 3956-2008" rules : $20^{\circ} \mathrm{C}$ conductance coefficient of copper is $57 \mathrm{~m} /\left(\Omega \cdot \mathrm{mm}^{2}\right)$, the DC resistance per unit length is $0.0731 \Omega / \mathrm{km}$. Considering the factor of temperature coefficient, skin effect and proximity effect coefficient, the AC resistance per unit length is 0.0911 $\Omega / \mathrm{km}$; ignore the inductance and capacitance of the cable; the cross of the zero line is half of the phase line.

The load power factor is 0.85 , considering the most serious situation that is running with full load. The load impedance is $Z=0.34+i 0.2107$ in which phase the load is the maximum phase.

\section{A. Distribution System Normal Operation with Three-Phase Load Unbalanced}

Analysis conditions: considering the load of phase B and $\mathrm{C}$ is equal to the full load, when the load umbalance degree of phase A , that is $k=\left(S_{C}-S_{A}\right) / S_{C}$ is $0 \sim 0.5$, the three-phase load voltage, displacement voltage $\mathrm{U}_{0}$, zero line current $3 \mathrm{I}_{0}$ are shown as Table.1.

TABLE I . LOAD VOLTAGE, ZERO-SEQUENCE VOLTAGE AND CURRENT WHEN NORMAL CONDITION WITH LOAD UNBALANCE.

\begin{tabular}{ccccccc}
\hline $\mathbf{k}$ & $\mathbf{0}$ & $\mathbf{0 . 1}$ & $\mathbf{0 . 2}$ & $\mathbf{0 . 3}$ & $\mathbf{0 . 4}$ & $\mathbf{0 . 5}$ \\
\hline UA & 220.2 & 222.9 & 225.7 & 228.5 & 231.4 & 234.3 \\
\hline UB & 220.2 & 219.9 & 219.7 & 219.5 & 219.4 & 219.2 \\
\hline UC & 220.2 & 218.8 & 217.4 & 215.9 & 214.3 & 212.8 \\
\hline U0 & 0 & 1.873 & 3.792 & 5.76 & 7.78 & 9.85 \\
\hline 3I0 & 0 & 41.11 & 83.25 & 126.4 & 170.7 & 216.1
\end{tabular}

The Table.1 shows that: when distribution system normal operation with three-phase load unbalanced, because the resistance of zero line is very small, the displacement voltage $\mathrm{U}_{0}$ is also small, less than safe voltage $36 \mathrm{~V}$, the load voltage is in the range of $10 \%$ rated voltage. When the load unbalance is more, the zero sequence current is larger.

\section{B. Zero Line Disconnection Occurs in Distribution System}

(1) There is only single-phase load after zero line disconnection

When zero line disconnection occurs at point"a"as in Figure.1, To Phase C, the voltage of phase line and zero line is equal, but with no current through load $\mathrm{C}$, the load can not work properly. The zero-sequence voltage is $230 \mathrm{~V}$.

(2) There is double phase load after zero line disconnection 
Analysis conditions: the double phase load are phase B and phase $\mathrm{C}$, considering the load of phase $\mathrm{C}$ is equal to the full load, when the load umbalance degree of phase $\mathrm{B}$, that is $k=\left(S_{C}-S_{B}\right) / S_{C}$ is $0 \sim 0.5$, the load voltage of phase B and C, displacement voltage $\mathrm{U}_{0}$ are shown as Table. 2.

TABLE II . LOAD VOLTAGE AND ZERO-SEQUENCE VOLTAGE WHEN DOUBLE-PHASE LOAD BEHIND THE ZERO LINE DISCONNECTION.

\begin{tabular}{ccccccc}
\hline $\mathbf{k}$ & $\mathbf{0}$ & $\mathbf{0 . 1}$ & $\mathbf{0 . 2}$ & $\mathbf{0 . 3}$ & $\mathbf{0 . 4}$ & $\mathbf{0 . 5}$ \\
\hline $\mathrm{U}_{\mathrm{B}}$ & 190.7 & 201 & 212.4 & 225.3 & 239.8 & 256.2 \\
\hline $\mathrm{U}_{\mathrm{C}}$ & 190.7 & 180.9 & 170 & 157.7 & 143.9 & 128.2 \\
\hline $\mathrm{U}_{0}$ & 112.6 & 113.2 & 114.8 & 118 & 122.9 & 130 \\
\hline
\end{tabular}

The Table.2 shows that: when there is double phase load (phase B and C) after zero line disconnection, the load voltage is relation to the load unbalance. The larger the load is, the lower the voltage. The load voltage of phase $\mathrm{C}$ in which phase the load is larger is not in the range of $\pm 10 \%$ rated voltage, the load can not work properly, and may damage the equipment. The displacement voltage $\mathrm{U}_{0}$ is more than safe voltage $36 \mathrm{~V}$, at least $112.6 \mathrm{~V}$.

(3) There is three phase load after zero line disconnection

Analysis conditions: considering the load of phase B and $\mathrm{C}$ is equal to the full load, when the load umbalance degree of phase $\mathrm{A}$, that is $k=\left(S_{C}-S_{A}\right) / S_{C}$ is $0 \sim 0.5$, the threephase load voltage, displacement voltage are shown as Table. 3.

\section{TABLE III . LOAD VOLTAGE AND ZERO-SEQUENCE VOLTAGE WHEN THREE -PHASE LOAD BEHIND THE ZERO LINE} DISCONNECTION.

\begin{tabular}{ccccccc}
\hline $\mathbf{k}$ & $\mathbf{0}$ & $\mathbf{0 . 1}$ & $\mathbf{0 . 2}$ & $\mathbf{0 . 3}$ & $\mathbf{0 . 4}$ & $\mathbf{0 . 5}$ \\
\hline UA & 220.2 & 228.5 & 237.6 & 247.3 & 257.9 & 269.2 \\
\hline UB & 220.2 & 216.5 & 212.8 & 209 & 205.3 & 201.6 \\
\hline UC & 220.2 & 216.8 & 213.4 & 209.9 & 206.5 & 203 \\
\hline U0 & 0 & 8.12 & 16.42 & 25.33 & 35.03 & 44.99
\end{tabular}

The Table.3 shows that: when there is three phase load after zero line disconnection, the load voltage is only relation to the three phase load unbalance. That is: the larger the load is, the lower the voltage is; and the more the load unbalance is, the greater the displacement voltage of neutral point is. When the load umbalance degree of phase $\mathrm{A}$ is more than $30 \%$, the load voltage of phase $\mathrm{A}$ is not in the range of $10 \%$ rated voltage, it can not work properly.

\section{PRotection Scheme}

Theoretical analysis and example calculation show that: for TN systems, when the zero line disconnection occurs, the zero line voltage is characterized as follows:

There is only single-phase load after the zero line disconnection: the zero line voltage after the zero line disconnection equals to the phase voltage. There are double phase load behind the zero line disconnection: the zero line voltage after the zero line disconnection is related to the load unbalance, the minimum is half of phase voltage, that is, $110 \mathrm{~V}$.
There are three phase loads after the zero line disconnection: the displacement voltage of neutral point is related to unbalance of three phase load.

The traditional method to obtain the zero line voltage is as follows. At the source of distribution system, there are three capacitors in $\mathrm{Y}$ connection which have equal capacity. Because the same capacity, the neutral point voltage of capacitor is zero. Then we can obtain the displacement voltage by measuring the voltage between the capacitor neutral point and the load neutral point. This method need to install capacitance and line. Considering the economy, this paper presents a new method to obtain zero sequence voltage.

Define that: $\dot{U}_{A 0}, \dot{U}_{B 0}, \dot{U}_{C 0}$ is Respectively the load voltage of phase $A, B, C$.

The zero sequence differential voltage is:

$$
\begin{aligned}
\dot{U}_{0}^{\prime} & =\frac{-\left(\dot{U}_{A 0}+\dot{U}_{B 0}+\dot{U}_{C 0}\right)}{3} \\
& =\dot{U}_{0}-\frac{\dot{U}_{A}+\dot{U}_{B}+\dot{U}_{C}}{3}
\end{aligned}
$$

$\dot{U}_{\text {unb }}=\frac{\dot{U}_{A}+\dot{U}_{B}+\dot{U}_{C}}{3}$ is the unbalance voltage. Because the impedance of phase line is very small, so the unbalance voltage $\dot{U}_{\text {unb }}$ is very less, The zero sequence differential voltage is Approximate equal to the voltage between the neutral point of zero line and the earth.

In order to prevent the protection mal-operation caused by the most load unbalance, the protection with zero sequence current blocking. The protection can act only when there is no zero sequence current and the zero sequence differential voltage is more than the setting.

The protection criterion: $\left|\dot{U}_{0}^{\prime}\right| \geq U_{\text {set }}$ 且 $\left|\dot{\mathrm{I}}_{0}\right| \leq I_{\text {set }}$

The setting of zero sequence differential voltage $U_{\text {set }}$ : Considering that the single-phase load can operate in the range of $10 \%$ rated voltage. The setting is according to $10 \%$ of the rated voltage, that is $22 \mathrm{~V}$.

\section{CONCLUSION}

When the zero line disconnection occurs in three-phase distribution system, an displacement voltage will be appeared at the neutral point.The displacement voltage magnitude is only relationed to the load umbalance, which makes the neutral moves to the phase with the larger load, resulting in an unblance of three phase voltage. That is: the larger the load is, the lower the voltage is; and the more the load unbalance is, the greater the displacement voltage of neutral point is. Based on this characteristic, a new method to detect zero line disconnection is presented based on zero sequence voltage with zero line current blocking. This method is more reliable, an example prove that the method is feasible.

\section{REFERENCES}

[1] Geng Yi. power supply of industrial enterprise [M]. Beijing: Metallurgical Industry Press, 1985. 
[2] Chen Yufeng. Analysis and Control of Drift of Electric Potential of Neutral Point in Power Supply System of Three-phase Four-wire[J]. Electric Engineering, 2002(6): 44-46.

[3] Luo Ming. Study o n Potential Deviation of Nentral Point in Powersupply System of Three- phase Four- wire System[J]. Electric Switchgear, 2003, 41(3):36-37.

[4] Liu Yi-ping. Analysis of the Offset of the Neutral Point's Electric Potential and It's Protection[J]. Journal of Shanghai Second Polytechnic University, 2005 (3):31-36.

[5] Study on Detection and Protection of Zero Line Disconnection in Threephase Four-wire System[J], Electrical Gengineering, 2008(8):83-85.

[6] ZHU Xue -ling. The Protection of Exceeded Voltage for the Ground Wire and the Disconnected Wire in Three -phase, Four -wire Lower Supplies and Distribution System[J]. Journal of North China Institute of Water Conservancy and Hydroelectric Power, 2004,25(3):33-35.

[7] WANG Yong, LI Zhiyong, WANG Yongzhong. Research of Intelligent Protection Device Realizing the Negative Wire Broken Protection in Three- phase Four- wire Power Supplying System[J]. Low Voltage Apparatus,2011(5):18-22.

[8] Yin Zhongdong. A detection device for the zero line disconnection in a three-phase four line system [P]. Chinese patent:200510086565, 200603-15.

[9] Luo Yi. A distributed online monitoring method and device for zero line disconnection [P] . Chinese patent:201010220891, 2011-01-19. 